Cahiers $d u$ MONDE RUSSE

\section{Cahiers du monde russe}

Russie - Empire russe - Union soviétique et États indépendants

$52 / 4 \mid 2011$

Varia

\title{
Wendy Z. Goldman, Inventing the Enemy
}

\section{François-Xavier Nérard}

\section{OpenEdition \\ Journals}

Édition électronique

URL : http://journals.openedition.org/monderusse/7620

DOI : $10.4000 /$ monderusse. 7620

ISSN : 1777-5388

Éditeur

Éditions de l'EHESS

\section{Édition imprimée}

Date de publication : 20 décembre 2011

Pagination : 740-743

ISBN : 978-2-7132-2353-2

ISSN : $1252-6576$

\section{Référence électronique}

François-Xavier Nérard, « Wendy Z. Goldman, Inventing the Enemy », Cahiers du monde russe [En ligne], 52/4 | 2011, mis en ligne le 30 novembre 2012, Consulté le 25 septembre 2020. URL : http:// journals.openedition.org/monderusse/7620 ; DOI : https://doi.org/10.4000/monderusse.7620

Ce document a été généré automatiquement le 25 septembre 2020

〔c École des hautes études en sciences sociales 


\title{
Wendy Z. Goldman, Inventing the Enemy
}

\author{
François-Xavier Nérard
}

\section{RÉFÉRENCE}

Wendy Z. GoLDMAN, Inventing the Enemy. Denunciation and Terror in Stalin's

Russia. Cambridge - New York : Cambridge University Press, 2011, 320 p.

1 Wendy Goldman poursuit ici la réflexion entamée dans son précédent ouvrage, Terror and Democracy in the Age of Stalin : the Social Dynamics of Repression ${ }^{1}$. Il s'agit pour elle de réfléchir aux dimensions sociales de la terreur stalinienne et à la participation populaire dans les événements de 1934-1938 en URSS. La démarche se veut explicitement «révisionniste »: la terreur pour Goldman n'est pas un phénomène uniquement décidé d'en haut. Son travail se fonde ainsi sur une approche large du phénomène. Plutôt que de la définir "d'en haut ", comme une succession de décisions politiques et policières, Goldman privilégie une approche de la «terreur» fondée sur l'expérience de la population: ce qui est vu, entendu, vécu et compris, même imparfaitement, par les Soviétiques. C'est ce choix qui dicte l'emploi du simple mot terror plutôt que Grande Terreur ou Ežovšina (p. 17-19). Son a priori est donc celui d'une histoire qui délaisse les « high politics» (p. 10) pour s'intéresser aux perceptions des simples Soviétiques, à leurs actes, à leur expérience. Elle aspire, grâce à cette approche, à nuancer les conclusions récentes de l'historiographie qui insistent notamment sur les opérations spéciales qui débutent pendant l'été 1937 avec l'ordre opérationnel 00447. Le rôle central de Stalin et des plus hauts dirigeants du pays (Ežov, Molotov, Kaganovič notamment), dans la conception et dans la mise en œuvre de la répression, a été ainsi bien mis en valeur ${ }^{2}$. Wendy Goldman choisit plutôt d'insister sur le rôle de la population dans cette terreur. Stalin et les dirigeants du parti n'auraient pas été les «seuls responsables de la terreur» (p. 80). Loin d'être de simples victimes passives, les Soviétiques auraient «aidé, à la fois individuellement et collectivement, à créer le tourbillon dans lequel ils étaient pris » (p. 136). De façon plus surprenante, elle semble 
également dire que la terreur aurait dépassé ses concepteurs et frappé «bien au-delà de ses victimes visées » (p. 191).

2 Pour mener à bien cette démonstration, Goldman choisit d'étudier les dénonciations de la population, sur une période qui couvre essentiellement les années 1936-1938: la montée en puissance du phénomène daterait du premier grand procès de Moscou (Goldman évoque une "fièvre croissante de dénonciations ", p. 1) et la décrue serait amorcée par la résolution de janvier 1938 du comité central (p. 260). Les dénonciations, orales et écrites sont ainsi considérées comme la forme de participation la plus répandue de la population à la terreur des années trente. Il faut saluer cette étude, la première aussi fouillée en anglais sur les dénonciations. Sheila Fitzpatrick, auparavant, avait dans quelques articles déjà travaillé sur la pratique ${ }^{3}$, mais Wendy Goldman fournit un travail plus extensif, bien différent dans sa méthode.

3 Elle se fonde sur des sources assez originales puisqu'il s'agit de comptes-rendus sténographiques de réunions des membres du parti communiste (VKP $[\mathrm{b}])$, tenues dans cinq usines de la région de Moscou entre 1934 et 1939 (Dinamo, Serp i Molot, Trehgornaja manufaktura, Krasnyj Poletarij et Likerno-vodočnyj zavod). Les lettres de dénonciations, elles-mêmes, sont assez peu mobilisées. Plutôt que de proposer une véritable étude des dénonciations en tant que telles (leurs formes, leurs contenus, leurs auteurs), Goldman privilégie les exemples en multipliant les études de cas. L'ouvrage délaisse ainsi volontiers la théorie et la généralisation pour raconter le destin d'individus broyés par la terreur. Le quatrième chapitre, malgré un titre singulier («Love, Loyalty and Betrayal»), permet ainsi de saisir les mécanismes implacables de la répression sur l'exemple de trois Soviétiques, employés à l'usine Serp i Molot. Cette attention portée aux individus donne à ce livre une teinte remarquable, qui fait que l'on ne sort pas indemne de cette lecture. Les comptes-rendus sténographiques constituent en outre une source très "vivante » : les larges extraits des échanges cités dans le livre contribuent également à rendre la terreur humaine. Ce type de sources a donc ses avantages, il a également ses inconvénients: il conduit à privilégier les dénonciations orales et à négliger l'écrit. Goldman mentionne et étudie à plusieurs reprises des lettres de dénonciation, mais elles ne sont pas au cœur de son travail. Au bilan, le livre ne tient pas toutes ses promesses et plutôt qu'un livre sur les dénonciations, il s'agit plutôt d'une étude de l'expérience populaire de la terreur, ce qui reste très intéressant, mais n'est pas exactement la même chose.

4 L'ouvrage est construit autour de cinq chapitres d'inégal intérêt. Le premier resitue la pratique de la dénonciation dans le cadre politique général des répressions. Le deuxième, le plus intéressant, porte sur l'étude de l'usine Dinamo pendant la période de la terreur. Il constitue un essai de microhistoire assez passionnant. Dans un troisième chapitre, Wendy Goldman ne s'enferme pas dans la question de destruction de la solidarité familiale par des dénonciations, mais tente d'étudier les conséquences de la terreur sur les liens familiaux. Elle montre avec beaucoup de nuances les difficultés et l'immense variété des positionnements. Le quatrième chapitre étudie la façon dont la terreur, à partir de 1937, prend pour cible les relations sociales et interpersonnelles et le cinquième, plus rapide, évoque la fin de la période des grandes répressions. Ces études ouvrent un certain nombre de débats :

5 - Le choix de se concentrer sur la période 1936-1938 est compréhensible. Pour autant, le manque de profondeur chronologique est, sur ce sujet précis des dénonciations, dommageable. On ne peut pas considérer qu'elles apparaissent véritablement en 1936, 
même s'il est certain que la seconde partie des années trente constitue un apogée du phénomène. Les méthodes, les pratiques, et même la "gigantesque machine linguistique " (p. 25) à juste titre étudiée, trouvent leurs racines bien des années auparavant. Les dénonciations de la terreur s'inscrivent dans une continuité. Elles ne sont pas, pour beaucoup, spécifiques à cette période.

6 - L'auteur ne parvient pas toujours à éviter les topoi de la littérature sur les dénonciations. C'est notamment vrai sur l'ampleur du phénomène, jamais quantifiée précisément, mais amplement décrite par des mots impressionnants et récurrents: flurry, ectasy of denunciations (p. 92). Le style est parfois déroutant, mobilisant des images peu rigoureuses: "flood, waves that rolled from Kharkov to Moscow, engulfing relatives». Il est certes bien difficile d'évaluer le nombre des dénonciations. Pour autant, Wendy Goldman a tendance à agréger des pratiques bien différentes : peut-on comparer la dénonciation d'un responsable insoupçonné et tout puissant, la dénonciation d'un homme déjà affaibli et soumis à la vindicte populaire ou celle de quelqu'un qui a déjà été arrêté (p. 301) ? Le contexte, les liens entre la victime et son dénonciateur méritent d'être plus étudiés qu'ils ne le sont.

7 - Wendy Goldman utilise plusieurs fois le terme de "coworkers", voire celui de «collègues» ou de «side-by-side» pour désigner les acteurs de la dénonciation. Elle n'aborde ainsi jamais explicitement la dimension sociale de la dénonciation stalinienne. Reprenant l'idée d'une dénonciation de tous par tous, elle néglige, alors que les très nombreux exemples qu'elle cite sont criants, le fait que ce sont d'abord et avant tout les cadres politiques et industriels du stalinisme qui sont visés par les dénonciations de la terreur, comme c'était déjà le cas, avec moins d'ampleur, auparavant. La dénonciation est majoritairement verticale. Bien entendu, des exemples subsistent de collègues de bureau qui se dénoncent, mais ils ne sauraient masquer la nature du phénomène.

- La question de la place de la dénonciation dans le processus répressif est laissée de côté, grâce à l'affirmation, pertinente, que « la réponse complète à cette question est encore cachée dans les archives » (p.138), même si Goldman en conclusion est plus affirmative, estimant que la plupart des dénonciations provoquaient « des enquêtes et des arrestations». Elle mérite cependant d'être posée. Les exemples donnés tout au long de l'ouvrage ne contribuent pas véritablement à donner cette impression. Les logiques de la répression nous semblent relativement indépendantes des dénonciations: c'est plus la répression qui provoque la dénonciation que les dénonciations qui sont à l'origine de la répression. Goldman ne contredit pas cette idée, mais ne conclut pas non plus en ces termes, laissant le lecteur quelque peu sur sa faim.

9 Le cœur de l'ouvrage est finalement plus une réflexion sur le comportement des individus face à une violence dont ils peinent à comprendre les enjeux et les logiques. La terreur des années 1936-1938 réduit à néant ou presque les marges d'autonomie de l'individu, qui doit plus ou moins s'adapter à ce que les autorités attendent de lui. C'est justement cette question de l'autonomie qui est centrale par rapport à l'hypothèse de Goldman du rôle des individus dans la terreur. Ont-ils le choix? Le dur chapitre 4 montre bien les marges de manœuvre extrêmement limitées, la faible importance d'une résistance à la pression ou à la torture.

Cette étude, importante, souvent nuancée, offre une approche intéressante de la terreur stalinienne. La question de la participation populaire à la terreur est essentielle, y compris dans la façon dont la Russie doit réfléchir à son passé. La dénonciation est un élément central de cette participation : Wendy Goldman entretient grâce à cet ouvrage 
un débat nécessaire. On regrettera peut-être qu'elle ne soit pas plus sensible à l'historiographie internationale sur les dénonciations, y compris, osons le dire, française ${ }^{4}$.

\section{NOTES}

1. Wendy Z. Goldman, Terror and Democracy in the Age of Stalin: the Social Dynamics of Repression, Cambridge - New York : Cambridge University Press, 2007.

2. Voir par exemple Nicolas Werth, L'ivrogne et la marchande de fleurs : Autopsie d'un meurtre de masse : 1937-1938, P. : Tallandier, 2009, Paul R. Gregory, Terror by Quota, New Haven : Yale University Press, 2009, ou Mark Junge, Rolf Binner, Kak terror stal "bol'šim”": sekretnyj prikaz n 00447 $i$ tehnologija ego ispolnenija [Comment la terreur est devenue "grande" : le décret secret n 00447 et la technologie de son application], Moscou : Airo-XX, 2003.

3. Sheila Fitzpatrick, Robert Gellately, Accusatory Practises: Denunciation in Modern European History, 1789-1989, Chicago : University of Chicago Press, 1997.

4. François-Xavier Nérard, Cinq pour cent de vérité, P. : Tallandier, 2004. 